Probability, Networks and Algorithms and Related Processes

J. van den Berg, O. Häggström, J. Kahn 
CWI is the National Research Institute for Mathematics and Computer Science. It is sponsored by the Netherlands Organization for Scientific Research (NWO).

$\mathrm{CWI}$ is a founding member of ERCIM, the European Research Consortium for Informatics and Mathematics.

CWI's research has a theme-oriented structure and is grouped into four clusters. Listed below are the names of the clusters and in parentheses their acronyms.

\section{Probability, Networks and Algorithms (PNA)}

Software Engineering (SEN)

Modelling, Analysis and Simulation (MAS)

Information Systems (INS)

Copyright (C) 2004, Stichting Centrum voor Wiskunde en Informatica

P.O. Box 94079, 1090 GB Amsterdam (NL)

Kruislaan 413, 1098 SJ Amsterdam (NL)

Telephone +31205929333

Telefax +31205924199

ISSN 1386-3711 


\title{
Some Conditional Correlation Inequalities for Percolation and Related Processes
}

\begin{abstract}
Consider ordinary bond percolation on a finite or countably infinite graph. Let $s, t$, $a$ and $b$ be vertices. An earlier paper proved the (nonintuitive) result that, conditioned on the event that there is no open path from $s$ to $t$, the two events "there is an open path from $s$ to a" and "there is an open path from $s$ to $b$ " are positively correlated. In the present paper we further investigate and generalize the theorem of which this result was a consequence. This leads to results saying, informally, that, with the above conditioning, the open cluster of $s$ is conditionally positively (self-)associated and that it is conditionally negatively correlated with the open cluster of t. We also present analogues of some of our results for (a) random-cluster measures, and (b) directed percolation and contact processes, and observe that the latter lead to improvements of some of the results in a paper of Belitsky, Ferrari, Konno and Liggett (1997).
\end{abstract}

2000 Mathematics Subject Classification: 60K35; 05C99; 60 C05

Keywords and Phrases: correlation inequalities; percolation; contact process; random-cluster model; Ahlswede-Daykin Theorem

Note: This work was carried out under project PNA3-General Research in Probability 


\title{
Some Conditional Correlation Inequalities for Percolation and Related Processes
}

\author{
J. van den Berg, O. Häggström and J. Kahn* \\ CWI, Chalmers University of Technology, and Rutgers University \\ email: J.van.den.Berg@cwi.nl; olleh@math.chalmers.se; jkahn@math.rutgers.edu
}

\begin{abstract}
Consider ordinary bond percolation on a finite or countably infinite graph. Let $s, t, a$ and $b$ be vertices. An earlier paper [3] proved the (nonintuitive) result that, conditioned on the event that there is no open path from $s$ to $t$, the two events "there is an open path from $s$ to $a$ " and "there is an open path from $s$ to $b$ " are positively correlated. In the present paper we further investigate and generalize the theorem of which this result was a consequence. This leads to results saying, informally, that, with the above conditioning, the open cluster of $s$ is conditionally positively (self-)associated and that it is conditionally negatively correlated with the open cluster of $t$.

We also present analogues of some of our results for (a) randomcluster measures, and (b) directed percolation and contact processes, and observe that the latter lead to improvements of some of the results in a paper of Belitsky, Ferrari, Konno and Liggett (1997).
\end{abstract}

\section{Introduction and results for ordinary per- colation}

This paper is concerned with positive and negative correlation and the stronger notion of positive association. Recall that events $A, B$ (in some probability

\footnotetext{
AMS 1991 subject classification: 05C99, 60C05, 60K35

Key words and phrases: correlation inequalities, percolation, contact process, randomcluster model, Ahlswede-Daykin Theorem

* Supported by NSF grant DMS0200856.
} 
space) are positively correlated if $\operatorname{Pr}(A B) \geq \operatorname{Pr}(A) \operatorname{Pr}(B)$, and negatively correlated if the reverse inequality holds. Positive association will be defined below (following Theorem 1.3).

We begin in this section with results for ordinary bond percolation. Our original motivation here (and for the present work) was Theorem 1.4.

We then consider extensions to the random cluster model (Section 2) and to percolation on directed graphs, together with applications to the contact process (Section 3).

A few words about proofs may be in order. The approach given for percolation in Section 1 is similar to that of [3] (see the proof of the present Theorem 1.1). This approach does not seem applicable to the random cluster model, and Section 2 takes a completely different route, based on Markov chains, to extend the results of Section 1 to this more general setting. We also describe, in Section 2.2, a different way of getting at some of the random cluster results. This is based on a connection with the "fuzzy Potts model," and is included here despite handling only a subset of what's covered by the Markov chain approach, because we think the relevance of the connection is interesting. The results for "directed percolation" in Section 3 can again be obtained using either the approach of Section 1 or the Markov chain approach of Section 2. Here we have tried to keep the discussion brief, mainly indicating those points where the generalization from what came before may not be entirely obvious.

Consider bond percolation on a (finite or countably infinite, locally finite) graph $G=(V, E)$, where each edge $e$ is, independently of all other edges, open with probability $p_{e}$ and closed with probability $1-p_{e}$. For $a, b \in V$ the event that there is an open path from $a$ to $b$ is denoted by $a \leftrightarrow b$, and the complement of this event by $a \nLeftarrow b$. For $X, Y \subset V$ we write $X \nLeftarrow Y$ for the event $\{x \nLeftarrow y \forall x \in X, y \in Y\}$.

In an earlier paper [3] we showed that, for any vertices $s, t, a, b$,

$$
\operatorname{Pr}(s \leftrightarrow a, s \leftrightarrow b \mid s \nleftarrow t) \geq \operatorname{Pr}(s \leftrightarrow a \mid s \nleftarrow t) \operatorname{Pr}(s \leftrightarrow b \mid s \nleftarrow t) .
$$

This was a consequence (really a special case) of Theorem 1.2 of [3], to which we will return below.

Here we show, among other results, a sort of complement of (1), viz.

$$
\operatorname{Pr}(s \leftrightarrow a, t \leftrightarrow b \mid s \nleftarrow t) \leq \operatorname{Pr}(s \leftrightarrow a \mid s \nleftarrow t) \operatorname{Pr}(t \leftrightarrow b \mid s \nleftarrow t) .
$$


In this section we will prove the quite intuitive (2) by way of a generalization of the not very intuitive (1). Before giving this generalization, we need some further definitions and notation.

Let $s$ be a fixed vertex. By the open cluster, $C_{s}$, of $s$ we mean the set of all edges which are in open paths starting at $s$. As in [3] we define, for $X \subseteq V$, the event

$$
R_{X}:=\{s \nLeftarrow X\}=\{s \nLeftarrow x \forall x \in X\} .
$$

Let $\Omega=\{0,1\}^{E}$ be the set of realizations; elements of $\Omega$ will typically be denoted $\omega$. Recall that an event $A$ is increasing (really, nondecreasing) if $\omega^{\prime} \geq \omega \in A$ implies $\omega^{\prime} \in A$. We also say that $A$ is increasing and determined by the open cluster of $s$ if $\omega \in A$ and $C_{s}\left(\omega^{\prime}\right) \supseteq C_{s}(\omega)$ imply $\omega^{\prime} \in A$. (Note that such an event is increasing in the sense above.) A simple example of such an event is $\{s \leftrightarrow a\}$.

The following statement is a natural generalization of Theorem 1.2 of [3].

Theorem 1.1. Let $A$ and $B$ be increasing events determined by the open cluster of $s$. Then for all $X, Y \subseteq V \backslash\{s\}$,

$$
\operatorname{Pr}\left(A R_{X}\right) \operatorname{Pr}\left(B R_{Y}\right) \leq \operatorname{Pr}\left(A B R_{X \cap Y}\right) \operatorname{Pr}\left(R_{X \cup Y}\right) .
$$

Remark. Theorem 1.2 in [3] is the special case where each of $A, B$ is of the form $\{s \leftrightarrow w \forall w \in W\}$ for some $W \subset V$. The proof of the present more general result is almost the same and we present it in a slightly abbreviated form, emphasizing the parts which need extra attention because of the generalization. (One may say that the key idea (in both cases) is generalizing from statements like (1) to the form (3), which supports an inductive proof.)

Proof. We give the proof for finite $G$; the infinite case then follows by standard limit arguments. The proof is by induction on the number of vertices. When $G$ has only one vertex, the result is obvious; so we suppose, for some $n \geq 1$, that the result holds for graphs with at most $n$ vertices, and consider $G$ with $n+1$ vertices.

With notation as in the theorem, it is easy to see that there is an event $\tilde{A} \subseteq A$ with the following properties: it is increasing and determined by $C_{s}$; it does not depend on

$$
E_{X}:=\{e \in E: e \cap X \neq \emptyset\}
$$


(that is, if $\omega_{e}^{\prime}=\omega_{e}$ for all $e \notin E_{X}$, then $\omega \in \tilde{A}$ iff $\omega^{\prime} \in \tilde{A}$ ); and, finally, $\tilde{A} R_{X}=A R_{X}$. A similar remark holds for $B$ and $Y$. So we may assume that $A$ does not depend on $E_{X}$ and $B$ does not depend on $E_{Y}$.

If $X \cap Y=\emptyset$, the r.h.s. of (3) is $\operatorname{Pr}(A B) \operatorname{Pr}\left(R_{X \cup Y}\right)$, and two applications of the FKG Inequality give the result:

$$
\operatorname{Pr}\left(A R_{X}\right) \operatorname{Pr}\left(B R_{Y}\right) \leq \operatorname{Pr}(A) \operatorname{Pr}(B) \operatorname{Pr}\left(R_{X}\right) \operatorname{Pr}\left(R_{Y}\right) \leq \operatorname{Pr}(A B) \operatorname{Pr}\left(R_{X \cup Y}\right)
$$

(note $R_{X \cup Y}=R_{X} R_{Y}$ ).

Now suppose $Z:=X \cap Y \neq \emptyset$. Let $N$ be the set of all vertices outside $Z$ with at least one neighbor in $Z$. Let $\mathbf{S}$ be the (random) set of those vertices of $N$ connected to $Z$ by at least one open edge. We have

$$
\operatorname{Pr}\left(A R_{X}\right)=\sum_{S} \operatorname{Pr}(\mathbf{S}=S) \operatorname{Pr}\left(A R_{X} \mid S\right)
$$

where the sum is over $S \subseteq N$ and we write $\operatorname{Pr}(\cdot \mid S)$ for $\operatorname{Pr}(\cdot \mid \mathbf{S}=S)$. Similar expressions hold for the other terms in (3). Moreover, clearly,

$$
\operatorname{Pr}(\mathbf{S}=S) \operatorname{Pr}(\mathbf{S}=T)=\operatorname{Pr}(\mathbf{S}=S \cap T) \operatorname{Pr}(\mathbf{S}=S \cup T) \quad \forall S, T \subseteq N .
$$

So according to the Ahlswede-Daykin ("Four Functions") Theorem ([1] or e.g. [4]), (3) will follow if we show that, for all $S, T \subseteq N$,

$$
\operatorname{Pr}\left(A R_{X} \mid S\right) \operatorname{Pr}\left(B R_{Y} \mid T\right) \leq \operatorname{Pr}\left(A B R_{X \cap Y} \mid S \cap T\right) \operatorname{Pr}\left(R_{X \cup Y} \mid S \cup T\right) .
$$

Now it is easy to see that, for any set of vertices $W \supseteq Z$, and any event $D$ that does not depend on $E_{W}$,

$$
\operatorname{Pr}\left(D R_{W} \mid S\right)=\operatorname{Pr}^{\prime}\left(D R_{(W \backslash Z) \cup S}\right),
$$

where $\operatorname{Pr}^{\prime}$ refers to the induced model on the graph $G^{\prime}$ obtained from $G$ by removing $Z$. (Strictly speaking, the $D$ on the r.h.s. of (6) is not the same as that on the 1.h.s., since it is a subset of $\{0,1\}^{E \backslash E_{Z}}$ rather than $\{0,1\}^{E}$; but since $D$ does not depend on $E_{W}$ (and hence not on $E_{Z}$ ), the two events are essentially the same, so we ignore the irrelevant distinction.)

Applying (6) to each of the four terms in (5), we have 


$$
\begin{aligned}
\operatorname{Pr}\left(A R_{X} \mid S\right) \operatorname{Pr}\left(B R_{Y} \mid T\right) & =\operatorname{Pr}^{\prime}\left(A R_{(X \backslash Z) \cup S}\right) \operatorname{Pr}^{\prime}\left(B R_{(Y \backslash Z) \cup T}\right) \\
& \leq \operatorname{Pr}^{\prime}\left(A B R_{((X \backslash Z) \cup S) \cap((Y \backslash Z) \cup T)}\right) \operatorname{Pr}^{\prime}\left(R_{(X \backslash Z) \cup S \cup(Y \backslash Z) \cup T}\right) \\
& \leq \operatorname{Pr}^{\prime}\left(A B R_{((X \cap Y) \backslash Z) \cup(S \cap T)}\right) \operatorname{Pr}^{\prime}\left(R_{((X \cup Y) \backslash Z) \cup(S \cup T)}\right) \\
& =\operatorname{Pr}\left(A B R_{X \cap Y} \mid S \cap T\right) \operatorname{Pr}\left(R_{X \cup Y} \mid S \cup T\right),
\end{aligned}
$$

where the first inequality follows from our inductive hypothesis (applicable since $G^{\prime}$ has fewer vertices than $G$ ), and the second from

$$
((X \backslash Z) \cup S) \cap((Y \backslash Z) \cup T) \supseteq((X \cap Y) \backslash Z) \cup(S \cap T)
$$

and $(X \backslash Z) \cup S \cup(Y \backslash Z) \cup T=((X \cup Y) \backslash Z) \cup(S \cup T)$.

In particular we have the promised generalization of (1):

Theorem 1.2. For $s, A, B$ and $X$ as in Theorem 1.1,

$$
\operatorname{Pr}(A B \mid s \nLeftarrow X) \geq \operatorname{Pr}(A \mid s \nLeftarrow X) \operatorname{Pr}(B \mid s \nLeftarrow X) .
$$

Proof. Take $Y=X$ in Theorem 1.1.

\section{Remarks}

1. It is easy to see that Theorem 1.2 is equivalent to the special case where $|X|=1$. (To reduce to this, simply identify the vertices of $X$, retaining all edges connecting them to $V \backslash X$ (edges internal to $X$ may be deleted, but are anyway irrelevant).) We have used the present form both because it will be convenient for the proof of Theorem 1.5, and because it is natural from the point of view of the contact process application in Section 3. Similarly, we could replace $s$ in all results of this section, and $t$ in Theorems 1.4 and 1.5 , by sets of vertices. The same easy equivalence holds for the directed graph results of Section 3; but in the case of the random cluster measures of Section 2 the more general statements, while still true, do not seem to follow in the same way from their specializations.

2. The derivation of Theorem 1.2 may give the impression that it is less general than Theorem 1.1, but in fact the two are equivalent. To see this, first note that (consideration of appropriate complementary events shows that) Theorem 1.2 also holds when $A$ and $B$ are both decreasing rather than increasing, while the inequality (7) reverses if one of $A, B$ is increasing and the other decreasing. Thus for $A, B, X, Y$ as in Theorem 1.1, Theorem 1.2 
implies that conditioned on $R_{X \cap Y}$, each of the pairs $\left(A, R_{X \backslash Y}\right),\left(B, R_{Y \backslash X}\right)$ is negatively correlated, while each of $(A, B),\left(R_{X \backslash Y}, R_{Y \backslash X}\right)$ is positively correlated. So, writing $\operatorname{Pr}^{\prime}$ for our percolation measure conditioned on $R_{X \cap Y}$, we have (compare (4))

$$
\begin{aligned}
\operatorname{Pr}^{\prime}\left(A R_{X \backslash Y}\right) \operatorname{Pr}^{\prime}\left(B R_{Y \backslash X}\right) & \leq \operatorname{Pr}^{\prime}(A) \operatorname{Pr}^{\prime}\left(R_{X \backslash Y}\right) \operatorname{Pr}^{\prime}(B) \operatorname{Pr}^{\prime}\left(R_{Y \backslash X}\right) \\
& \leq \operatorname{Pr}^{\prime}(A B) \operatorname{Pr}^{\prime}\left(R_{X \backslash Y} R_{Y \backslash X}\right),
\end{aligned}
$$

which is equivalent to (3).

It will be helpful to have the "functional extension" of Theorem 1.2:

Theorem 1.3. For $s, X$ as in Theorem 1.2, and $f, g$ bounded, increasing, measurable functions of $C_{s}$,

$$
\mathrm{E}[f g \mid s \nLeftarrow X] \geq \mathrm{E}[f \mid s \nLeftarrow X] \mathrm{E}[g \mid s \leftrightarrow X]
$$

The inequality is reversed if one of $f, g$ is increasing and the other decreasing.

Proof. This is a standard (and easy) reduction. We omit the argument for (a) and note that (b) is (a) applied to the pair $(f,-g)$.

Recall that a collection of random variables $\left\{\sigma_{i}: i \in \Gamma\right\}$, with $\Gamma$ a finite or countably infinite index set and the $\sigma_{i}$ 's taking values in $\{0,1\}$ (or some other ordered set), is said to be positively associated if for any two functions $f, g$ of the $\sigma_{i}$ 's that are either both increasing or both decreasing (and, in case $\Gamma$ is infinite, measurable), one has $\mathrm{E} f g \geq \mathrm{E} f \mathrm{E} g$. The simplest non-trivial example is when the $\sigma_{i}$ 's are independent (Harris' inequality).

If we define a random subset $W$ of a set $T$ to be positively associated if the collection $\left\{\eta(a)=\mathbf{1}_{\{a \in W\}}: a \in T\right\}$ is positively associated, then Theorem 1.3 says that the open cluster of $s$ is conditionally positively associated given the event $\{s \nLeftarrow X\}$. We will see further, similar examples later.

Positive association is often derived from the FKG Inequality, which generalizes Harris' inequality and says that positive association holds for measures (on $\{0,1\}^{n}$, say) satisfying the "positive lattice condition" (also called "FKG lattice condition"), viz.

$$
\mu(\sigma) \mu(\tau) \leq \mu(\sigma \wedge \tau) \mu(\sigma \vee \tau)
$$


(where $(\sigma \wedge \tau)_{x}$ and $(\sigma \vee \tau)_{x}$ are the minimum and maximum of $\sigma_{x}$ and $\tau_{x}$ ). The positive lattice condition is much stronger than positive association. It says that the conditional probability that $\sigma_{x}=1$, given the values of $\sigma_{y}, y \neq x$, is increasing in those values.

Let us also recall here that for measures $\nu$ and $\nu^{\prime}$ on $\{0,1\}^{n}$ (or some other partially ordered set), $\nu$ stochastically dominates $\nu^{\prime}\left(\nu \succ \nu^{\prime}\right)$ if $\nu(f) \geq \nu^{\prime}(f)$ for every increasing function $f$ (where $\nu(f)$ is the expectation of $f$ w.r.t. $\nu$ ).

As suggested earlier, we do not see any good reason to expect something like Theorem 1.2. (For instance, as noted in [3], it is easy to see that the analogous statement with $s \leftrightarrow t$ in place of $s \nLeftarrow t$ is false.) Nonetheless, it implies the following intuitively more natural statement, which says, informally, that conditioned on nonexistence of an open $(s, t)$-path, the clusters $C_{s}$ and $C_{t}$ are negatively correlated.

Theorem 1.4. Let $s$ and $t$ be (distinct) vertices, and $f$ and $g$ bounded measurable increasing functions of $C_{s}$ and $C_{t}$ respectively. Then

$$
\mathrm{E}[f g \mid s \nLeftarrow t] \leq \mathrm{E}[f \mid s \nLeftarrow t] \mathrm{E}[g \mid s \nLeftarrow t] .
$$

Note that (2) is the special case where $f$ is the indicator of the event $\{s \leftrightarrow a\}$, and $g$ that of the event $\{t \leftrightarrow b\}$.

We have stated Theorem 1.4 above largely because, as mentioned earlier, it was the original motivation for this work; but the next statement, which contains Theorem 1.3 as well as Theorem 1.4, seems to be the correct level of generality here.

Theorem 1.5. Let $s$ and $t$ be (distinct) vertices, and $f$ and $g$ bounded, measurable functions of $\left(C_{s}, C_{t}\right)$, each increasing in $C_{s}$ and decreasing in $C_{t}$. Then

$$
\mathrm{E}[f g \mid s \nLeftarrow t] \geq \mathrm{E}[f \mid s \nLeftarrow t] \mathrm{E}[g \mid s \nLeftarrow t] .
$$

In other words, on $\{s \nLeftarrow t\}$ we have positive association of all the r.v.'s $\mathbf{1}_{\left\{e \in C_{s}\right\}}$ and $\mathbf{1}_{\left\{e \notin C_{t}\right\}}$. (Note: here and often in what follows, we use "on $Q$ " to mean "conditioned on (the event) $Q . "$ )

Proof of Theorem 1.5. As in the case of Theorem 1.1, it is enough to prove this for finite $G$.

We have

$$
\mathrm{E}[f g \mid s \nleftarrow t]=\sum_{W} \operatorname{Pr}\left(C_{s}=W \mid s \nleftarrow t\right) \mathrm{E}\left[f g \mid C_{s}=W\right],
$$


where we may restrict to $W$ containing no $(s, t)$-path. Write $\bar{W}$ for the union of $W$ and its "boundary"; that is, $\bar{W}$ consists of all edges having at least one vertex in common with some edge of $W$.

When we condition on $\left\{C_{s}=W\right\}, f$ and $g$ become decreasing functions of $C_{t}$, and the (conditional) distribution of $C_{t}$ is the same as that for the restriction of our percolation model to the graph obtained from $G$ by deleting all edges in $\bar{W}$. Thus (on $\left\{C_{s}=W\right\}$ ) $f, g$ are decreasing functions of the independent r.v.'s $\left(\omega_{e}: e \in E \backslash \bar{W}\right)$, and by Harris' inequality we have $\mathrm{E}\left[f g \mid C_{s}=W\right] \geq \mathrm{E}\left[f \mid C_{s}=W\right] \mathrm{E}\left[g \mid C_{s}=W\right]$.

On the other hand, the conditional distribution of $C_{t}$ given $\left\{C_{s}=W\right\}$ is stochastically decreasing in $W$ (to couple these distributions, choose all $\omega_{e}$ 's independently according to their $p_{e}$ 's and then for conditioning on $\left\{C_{s}=\right.$ $W\}$ simply ignore those $\omega_{e}$ 's with $\left.e \in \bar{W}\right)$; so in particular $\mathrm{E}\left[f \mid C_{s}=W\right]$ and $\mathrm{E}\left[g \mid C_{s}=W\right]$ are increasing functions of $W$, and it then follows from Theorem 1.3 that the right hand side of (10) is not less than

$$
\begin{gathered}
\left(\sum_{W} \operatorname{Pr}\left(C_{s}=W \mid s \nLeftarrow t\right) \mathrm{E}\left[f \mid C_{s}=W\right]\right)\left(\sum_{W}\left[\operatorname{Pr}\left(C_{s}=W \mid s \nLeftarrow t\right) \mathrm{E}\left[g \mid C_{s}=W\right]\right)\right. \\
=\mathrm{E}[f \mid s \nLeftarrow t] \mathrm{E}[g \mid s \nLeftarrow t] ;
\end{gathered}
$$

so we have (9).

As just shown, Theorem 1.4 follows easily from Theorem 1.3. While one might expect a similar proof (or some proof) of the reverse implication, we do not see this. In Section 2 we will (as mentioned earlier) take a completely different approach which, even for the more general class of random-cluster measures, gives Theorems 1.3 and 1.4 "simultaneously".

\section{Random-cluster measures}

\subsection{Definitions and a Markov chain proof}

A well-known generalization of the bond percolation model is the randomcluster model (RCM) introduced by Fortuin and Kasteleyn circa 1970. (See e.g. [6], Section 13.6, [7] for additional background and references.)

Let $G=(V, E)$ be a finite graph. In addition to the parameters $p_{e}, e \in$ $E$ of the ordinary bond percolation model, the random-cluster model is 
equipped with a positive parameter $q$. To avoid trivialities we assume that $0<p_{e}<1$ for all $e \in E$. The random-cluster measure (r.c.m.) with the above parameters on $\Omega=\{0,1\}^{E}$ is then given by

$$
\varphi_{q}(\omega)\left(=\varphi_{G, q}(\omega)\right) \propto q^{k(\omega)} \prod_{e \in E: \omega_{e}=1} p_{e} \prod_{e \in E: \omega_{e}=0}\left(1-p_{e}\right), \quad \omega \in \Omega
$$

where $k(\omega)$ is the number of connected components in $\omega$, and, as usual, $f(\omega) \propto g(\omega)$ means $f(\omega)=C g(\omega)$ for some (positive) constant $C$. (For the present discussion we regard the $p_{e}$ 's as given once and for all, and omit them from our notation.)

Thus $q=1$ gives the ordinary bond percolation model. We have, in spite of serious attempts, not been able to adapt the approach of Section 1 to $q>1$. (We do not consider $q<1$, for which the correlation properties of the model are quite different). Here we take a different, "dynamical" approach, based on the introduction of a Markov chain whose states are pairs of clusters (this is not the only possibility; see the remark following the proof of Theorem 2.1) which converges to a measure (on pairs of clusters) corresponding to (11), and for intermediate stages of which the correlation properties we are after can be derived from known properties of the RCM.

For the following extension of Theorem 1.5 to the RCM we replace the vertices $s$ and $t$ by sets $S$ and $T$, recalling that the remark following Theorem 1.2 regarding the easy reduction from sets to singletons is not valid here. Extending our earlier notation, we use $C_{S}$ for the set of edges belonging to open paths starting at vertices of $S$.

Theorem 2.1. Consider a distribution (11) with $q \geq 1$. Let $S$ and $T$ be disjoint sets of vertices, and $f$ and $g$ bounded, measurable functions of $\left(C_{S}, C_{T}\right)$, each increasing in $C_{S}$ and decreasing in $C_{T}$. Then on $\{S \nLeftarrow T\}$,

$$
\mathrm{E} f g \geq \mathrm{E} f \mathrm{E} g
$$

Following the Markov chain proof of this, we also give, in Section 2.2, a different argument, which unfortunately seems only to work when $q \geq 2$ and $|S|=|T|=1$. So, somewhat strangely, we have separate (and distinct) proofs for the cases $q=1$ and $q \geq 2$, but for the intermediate case $1<q<2$, no alternative to the Markov chain approach. 
Proof. We first give some additional notation, and state some (well-known) lemmas we will need. If $F$ is a subset of $E$ (the set of edges of our graph $G)$, and $\omega \in \Omega=\{0,1\}^{E}$, we write $\omega_{F}$ for the restriction of $\omega$ to $F\left(\omega_{F}=\right.$ $\left(\omega_{e}: e \in F\right)$ ), and $V(F)$ for the set of vertices incident with edges of $F$. We continue to use the notation $\bar{W}$ introduced following (10).

Lemma 2.2. For $q \geq 1$, the random-cluster measure (11) satisfies the positive lattice condition (8).

When $\varphi_{G, q}$ is conditioned on the values of some of the variables $\omega_{e}$, the remaining variables are distributed as they would be under the (natural) r.c.m. on the graph obtained from $G$ by deleting $e$ 's with $\omega_{e}=0$ and contracting those with $\omega_{e}=1$. For our purposes the relevant cases of this are given by

Lemma 2.3. Let $A \subset V$ and $F \subset E$. The restriction of $\varphi_{G, q}$ to $\left.\{0,1\}\right\}^{E \backslash \bar{F}}$ under conditioning on either of the events $\left\{C_{A}=F\right\},\left\{\omega_{\bar{F}} \equiv 0\right\}$ (i.e. $\left\{\omega_{e}=\right.$ $0 \forall e \in \bar{F}\}$ ) is the r.c.m. with parameter $q$ on $G-\bar{F}$ (the graph obtained from $G$ by deleting all edges in $\bar{F})$; more formally,

$$
\varphi_{G, q}\left(\omega_{E \backslash \bar{F}}=\cdot \mid C_{A}=F\right)=\varphi_{G, q}\left(\omega_{E \backslash \bar{F}}=\cdot \mid \omega_{\bar{F}} \equiv 0\right)=\varphi_{G-\bar{F}, q}(\cdot) .
$$

If $A, F$ are as in Lemma 2.3, and $B \subseteq V \backslash V(F)$, then $\left\{C_{A}=F\right\} \subseteq\{A \nleftarrow B\}$; so Lemma 2.3 implies

Lemma 2.4. If $A, F$ are as in Lemma 2.3, $B \subseteq V \backslash V(F)$, and $\varphi$ is (temporarily) $\varphi_{G, q}$ conditioned on $\{A \leftrightarrow B\}$, then

$$
\varphi\left(\omega_{E \backslash \bar{F}}=\cdot \mid C_{A}=F\right)=\varphi_{G, q}\left(\omega_{E \backslash \bar{F}}=\cdot \mid \omega_{\bar{F}} \equiv 0\right)=\varphi_{G-\bar{F}, q}(\cdot) .
$$

We now turn to the proof of Theorem 2.1. We consider a Markov chain with state space $\hat{\Omega}$ consisting of pairs $\left(C_{S}, C_{T}\right)$ satisfying $Q:=\{S \nLeftarrow T\}$. (So the states are pairs $\left(C, C^{\prime}\right)$ such that $C, C^{\prime} \subseteq E ; C$ (resp. $\left.C^{\prime}\right)$ is a union of paths beginning at vertices of $S$ (resp. vertices of $T$ ); and $V(C) \cap V\left(C^{\prime}\right)=\emptyset$.)

We write $\varphi$ for the measure $\varphi_{G, q}$ conditioned on $Q$ and $\hat{\varphi}$ for the measure which $\varphi$ induces on $\hat{\Omega}$.

Initially our chain is in some fixed state $\left(C_{S}^{0}, C_{T}^{0}\right) \in \hat{\Omega}$. Given $\left(C_{S}^{i-1}, C_{T}^{i-1}\right)$, the state of the chain at time $i-1$, we choose $\left(C_{S}^{i}, C_{T}^{i}\right)$ in two steps, first choosing $C_{T}^{i}$ according to $\varphi$ conditioned on $\left\{C_{S}=C_{S}^{i-1}\right\}$ - that is,

$$
\operatorname{Pr}\left(C_{T}^{i}=\cdot\right)=\varphi\left(C_{T}=\cdot \mid C_{S}=C_{S}^{i-1}\right)
$$


—and then, similarly, $C_{S}^{i}$ according to

$$
\operatorname{Pr}\left(C_{S}^{i}=\cdot\right)=\varphi\left(C_{S}=\cdot \mid C_{T}=C_{T}^{i}\right) .
$$

It is clear that $\hat{\varphi}$ is stationary for this chain, and that the chain is irreducible and aperiodic; so to prove Theorem 2.1 it's enough to show

Claim 2.5. For $f, g$ as in the statement of Theorem 2.1 and any n, (12) holds for expectation taken with respect to the law of $\left(C_{S}^{n}, C_{T}^{n}\right)$.

Let $X_{e}^{i}$ and $Y_{e}^{i}$ be the indicators of the events $\left\{e \notin C_{T}^{i}\right\}$ and $\left\{e \in C_{S}^{i}\right\}(e \in E$, $i=0,1, \ldots)$. These are, of course, not independent, but we will show, using the following presumably well-known observation, that they are positively associated.

Lemma 2.6. Suppose $W_{1}, \ldots, W_{a}$ and $Z_{1}, \ldots, Z_{b}$ are (say) $\{0,1\}$-valued r.v.'s with joint distribution $\psi$ satisfying

(i) $W_{1}, \ldots, W_{a}$ are positively associated;

(ii) $Z_{1}, \ldots, Z_{b}$ are conditionally positively associated given $W_{1}, \ldots, W_{a}$; and

(iii) for $W, W^{\prime} \in\{0,1\}^{a}$ with $W^{\prime} \geq W, \psi\left(\cdot \mid W^{\prime}\right) \succ \psi(\cdot \mid W)$, where $\psi(\cdot \mid W)$ is the conditional distribution of $\left(Z_{1}, \ldots, Z_{b}\right)$ given $\left(W_{1}, \ldots, W_{a}\right)=W$.

Then $W_{1}, \ldots, W_{a}, Z_{1}, \ldots, Z_{b}$ are positively associated.

Proof. Suppose $f, g$ are increasing functions of $W_{1}, \ldots, Z_{b}$, and for $W \in$ $\{0,1\}^{a}$, set $F(W)=\mathrm{E}[f \mid W]\left(:=\mathrm{E}\left[f \mid\left(W_{1}, \ldots, W_{a}\right)=W\right]\right)$ and $G(W)=$ $\mathrm{E}[g \mid W]$. Then

$$
\begin{aligned}
\mathrm{E} f g & =\mathrm{E}\{\mathrm{E}[f g \mid W]\} \\
& \geq \mathrm{E}\{\mathrm{E}[f \mid W] \mathrm{E}[g \mid W]\} \\
& \geq \mathrm{E} F \mathrm{E} G \\
& =\mathrm{E} f \mathrm{E} g,
\end{aligned}
$$

where the first inequality follows from (ii) and the second from (iii) and (i).

Lemma 2.7. The collection

$$
\cup_{i \geq 1} \cup_{e \in E}\left\{X_{e}^{i}, Y_{e}^{i}\right\}
$$

is positively associated. 
Note this is enough for Claim 2.5 since (trivially)

Remark 2.8. For each $n, C_{S}^{n}$ is increasing in the variables $X_{e}^{i}, Y_{e}^{i}$, and $C_{T}^{n}$ is decreasing in these variables.

Proof of Lemma 2.7. Of course it's enough to show positive association for finite subsets of the collection (13). We will show by induction on $n$ that for each $n$, each of the collections

$$
\left\{X_{e}^{i}: e \in E, i \leq n\right\} \cup\left\{Y_{e}^{i}: e \in E, i<n\right\}
$$

and

$$
\left\{X_{e}^{i}: e \in E, i \leq n\right\} \cup\left\{Y_{e}^{i}: e \in E, i \leq n\right\}
$$

is positively associated. (The base cases - those with $n=0$-are, of course, trivial.) Actually we just give the argument for (14), that for (15) being essentially the same.

We want to apply Lemma 2.6 with $\left(W_{1}, \ldots, W_{a}\right)=\cup\left(\left(X_{e}^{i}, Y_{e}^{i}\right): e \in\right.$ $E, i<n)$ and $\left(Z_{1}, \ldots, Z_{b}\right)=\left(X_{e}^{n}: e \in E\right)$, so need to verify conditions (i)-(iii) of the lemma. Of course (i) is just our inductive hypothesis, so our concern is really with (ii) and (iii).

Consider a possible value $W$ of $\left(W_{1}, \ldots, W_{a}\right)$, with $F$ the corresponding value of $C_{S}^{n-1}$. Under conditioning on $\left\{\left(W_{1}, \ldots, W_{a}\right)=W\right\}$, we have $X_{e}^{n}$ fixed for $e \in \bar{F}$ (namely $X_{e}^{n} \equiv 1 \forall e \in \bar{F}$ ), while, by Lemma 2.4, the remaining $X_{e}^{n}$ 's are distributed as the variables $1_{\left\{e \notin C_{t}(\omega)\right\}}$, where $\left(\omega_{e}: e \in E \backslash \bar{F}\right)$ is chosen according to $\varphi_{G-\bar{F}, q}$. Positive association of these variables is given by Lemma 2.2, so we have (ii).

Now let $W^{\prime}$ be a second possible value of $\left(W_{1}, \ldots, W_{a}\right)$, with $W^{\prime} \geq W$ and $F^{\prime}$ the corresponding value of $C_{S}^{n-1}$. According to Remark 2.8 we have $F \subseteq F^{\prime}$. So (iii) amounts to saying that for $F \subseteq F^{\prime} \subseteq E$ and $h$ any increasing function of $C_{T}$,

$$
\varphi\left(h \mid C_{S}=F\right) \geq \varphi\left(h \mid C_{S}=F^{\prime}\right)
$$

(note $h$ is a decreasing function of the $X_{e}^{n}$ 's). But using Lemmas 2.3 and 2.4, we may rewrite the left and right hand sides of (16) as

$$
\varphi_{G-\bar{F}, q}(h)
$$

and

$$
\varphi_{G-\bar{F}^{\prime}, q}(h)=\varphi_{G-\bar{F}, q}\left(h \mid \omega_{\bar{F}^{\prime} \backslash \bar{F}} \equiv 0\right) ;
$$

and then (16) follows from Lemma 2.2 (which gives positive association for the measure $\left.\varphi_{G-\bar{F}, q}\right)$. 
Remark. We briefly indicate an alternative proof of Theorem 2.1, again using a Markov chain and based on a similar idea. This, our original proof, is perhaps more natural than that given above, but does not seem as easily adapted to prove the directed version of Theorem 1.5 (Theorem 3.4).

We again use $\varphi$ for $\varphi_{G, q}$ conditioned on $\{S \nLeftarrow T\}$. Our chain in this case is $\omega^{0}, \omega^{1}, \omega^{2}, \ldots$ drawn from the state space $\hat{\Omega}:=\{\omega \in \Omega: S \nLeftarrow T\}$. Initially the chain is in some fixed state $\omega^{0}$. Given $\omega^{i-1}$, the state of the chain at time $i-1$, we choose $\omega^{i}$ in two steps, first choosing an intermediate configuration $\tau^{i}$ according to $\varphi$ conditioned on $\left\{C_{S}=C_{S}\left(\omega^{i-1}\right)\right\}$ - that is, for $\zeta \in \hat{\Omega}$ with $C_{S}(\zeta)=C_{S}\left(\omega^{i-1}\right)$

$$
\operatorname{Pr}\left(\tau^{i}=\zeta\right)=\varphi\left(\omega=\zeta \mid C_{S}(\omega)=C_{S}\left(\omega^{i-1}\right)\right)
$$

—and then, similarly, $\omega^{i}$ according to

$$
\operatorname{Pr}\left(\omega^{i}=\zeta\right)=\varphi\left(\omega=\zeta \mid C_{T}(\omega)=C_{T}\left(\tau^{i}\right)\right) .
$$

It is clear that $\varphi$ is stationary for this chain, and that the chain is irreducible and aperiodic; so to prove Theorem 2.1 it's enough to show

Claim. For $f, g$ as in the statement of Theorem 2.1 and any n, (12) holds for expectation taken with respect to the law of $\left(\omega^{n}\right)$.

To prove this we introduce independent r.v.'s $X_{e}^{i}, Y_{e}^{i}(e \in E, i=1, \ldots)$, each

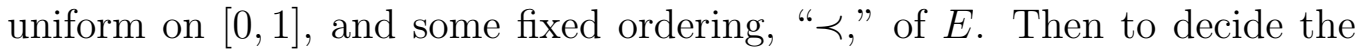
value of $\tau_{e}^{i}$ we compute the conditional probability, say $\alpha$, that $\tau_{e}^{i}=1$ given the values of the $\omega_{e}^{i-1}$ 's (or just the value of $C_{S}\left(\omega^{i-1}\right)$ ) and those $\tau_{e^{\prime}}^{i}$ 's with $e^{\prime} \prec e$, and set $\tau_{e}^{i}=1$ iff $X_{e}^{i}<\alpha$. For $\omega^{i}$ we proceed analogously, with the requirement for $\omega_{e}^{i}=1$ now being $Y_{e}^{i}>1-\alpha$.

It is then not hard to show, again using Lemmas 2.2-2.4, that (for each n) $\omega^{n}$ is increasing in the variables $X_{e}^{i}, Y_{e}^{i}$, so that the Claim follows from Harris' inequality.

\section{$2.2 \quad$ A separate proof for $q \geq 2$}

As mentioned earlier, it turns out, somewhat curiously, that for $q \geq 2$ and $S$ and $T$ consisting of single vertices $s$ and $t$, we can prove Theorem 2.1 in a different way by exploiting a connection between the random cluster model and the "fuzzy Potts model." (The corresponding connection involving the 
ordinary Potts model again goes back to Fortuin and Kasteleyn.) Before doing so, we need to review some classical and more recent facts concerning this connection.

Let $q=\alpha+\beta$ with $\alpha, \beta>0$. Using the random-cluster measure $\varphi$ we generate a random spin configuration $\sigma \in\{0,1\}^{V}$ as follows.

(i) Choose $\omega \in\{0,1\}^{E}$ according to $\varphi_{q}$.

(ii) For each component $C$ of $\omega$, let $\sigma$ take the value 1 (resp. 0) on all vertices of $C$ with probability $\alpha / q$ (resp. $\beta / q$ ), independently of the values of $\sigma$ on other components. Let $\mu_{\alpha, \beta}$ denote the distribution of $\sigma$.

(In [8] this is called the fractional fuzzy Potts model.) This procedure produces a coupling measure $\mathcal{P}$ of $\omega$ and $\sigma$, or, rather, of $\varphi_{q}$ and $\mu_{\alpha, \beta}$. So we may also think of first choosing $\sigma$ and then drawing from the conditional distribution $\mathcal{P}(\cdot \mid \sigma)$ to obtain a typical (with distribution $\varphi_{q}$ ) edge configuration $\omega$. It is known (and easy to check) that this "reversed" procedure can be described as follows.

(iii) Choose $\sigma$ according to $\mu_{\alpha, \beta}$.

(iv) For $i=1,0$, let $G(i)=G\left[\sigma^{-1}(i)\right]$ (the (induced) subgraph consisting of vertices in $\sigma^{-1}(i)$ and edges of $G$ contained in this set). Set $\omega_{e}=0$ whenever $\sigma$ assigns different values to the ends of $e \in E$, and choose the restrictions of $\omega$ to $E(G(1))$ and $E\left(G(0)\right.$ ) (independently) according to $\varphi_{G(1), \alpha}$ and $\varphi_{G(0), \beta}$.

Furthermore, if in (iii) we choose $\sigma$ according to the conditional distribution

$$
\hat{\mu}_{\alpha, \beta}(\cdot)=\mu_{\alpha, \beta}(\cdot \mid \sigma(s)=1, \sigma(t)=0),
$$

then $\omega$ (in (iv)) has the distribution we want, namely $\varphi_{G, q}(\cdot \mid s \nless t$ ); so for Theorem 2.1 we may take $\omega$ to be chosen in this way.

The salient points for our purposes are then as follows. (For clarity we now add subscripts to the expectation symbol $E$ to indicate measures with respect to which expectation is taken.)

(a) (Lemma 2.2) For any graph $H$ and $c \geq 1, \varphi_{H, c}$ has positive association.

(b) It is shown in [8] that for any $\alpha, \beta \geq 1, \mu=\mu_{\alpha, \beta}$ satisfies (8), whence, according to the FKG Inequality, $\mu_{\alpha, \beta}$ and $\hat{\mu}_{\alpha, \beta}$ are positively associated. 
(c) If $\alpha, \beta \geq 1$ and $f$ is a function of $\left(C_{s}, C_{t}\right)$ which is increasing in $C_{s}$ and decreasing in $C_{t}$, then $\mathrm{E}_{\mathcal{P}}[f \mid \sigma]$ is increasing $\sigma$. (This follows from ((iv) and) (a).)

Alternate proof of Theorem 2.1 for $q \geq 2, S=\{s\}$ and $T=\{t\}$.

Let $f, g$ be as in the statement of the theorem. Fix some $\alpha, \beta \geq 1$ with $\alpha+\beta=q$. For simplicity we write $\mu$ for $\mu_{\alpha, \beta}$ and $\varphi$ for $\varphi_{q}$. The connections described above give

$$
\begin{aligned}
\mathrm{E}_{\varphi}[f g \mid s \leftrightarrow t] & =\sum_{\sigma} \hat{\mu}(\sigma) \mathrm{E}_{\mathcal{P}}[f(\omega) g(\omega) \mid \sigma] \\
& \geq \sum_{\sigma} \hat{\mu}(\sigma) \mathrm{E}_{\mathcal{P}}[f(\omega) \mid \sigma] \mathrm{E}_{\mathcal{P}}[g(\omega) \mid \sigma] \\
& \geq \sum_{\sigma} \hat{\mu}(\sigma) \mathrm{E}_{\mathcal{P}}[f(\omega) \mid \sigma] \sum_{\sigma} \hat{\mu}(\sigma) \mathrm{E}_{\mathcal{P}}[g(\omega) \mid \sigma] \\
& =\mathrm{E}_{\varphi}[f \mid s \nLeftarrow t] \mathrm{E}_{\varphi}[g \mid s \nLeftarrow t],
\end{aligned}
$$

where the first inequality follows from (a) (and (iv)) and the second from (b) and $(c)$.

\section{Directed percolation and contact processes}

In this section we consider another generalization of ordinary percolation: as in Section 1 we have a product distribution on $\{0,1\}^{E}$, but now some (or all, or none) of the edges of our graph are oriented. There are (at least) two natural ways to try to extend the results of Section 1 to this setting, corresponding to two possible extensions of the conditioning event $\{s \not t t\}$. As we will see, both extensions are reasonable for Theorems 1.1-1.3, but only one of them makes sense for Theorem 1.4 (and Theorem 1.5). The first set of extensions yield in particular improvements of some of the results of Belitsky, Ferrari, Konno and Liggett [2] regarding the contact process (defined below). We will first indicate these extensions (proofs of which are essentially identical to the proofs of the corresponding statements in Section 1) and discuss their relevance to the contact process, before turning to the second set of extensions.

We will need the following additional notation. Unoriented edges will be denoted by $\{v, w\}$ and oriented edges by $(v, w)$ (where the orientation 
is from $v$ to $w$ ). When we speak of a path, we will now mean one which respects the orientations of its oriented edges. We write $\{s \rightarrow t\}$ for the event that there is an open path from $s$ to $t$ and $\{s \nrightarrow t\}$ for the complement of this event. The open cluster, $C_{s}$, of $s$ is again the set of all edges contained in open paths starting at $s$. As in Section 1, we fix a vertex $s$ and set $R_{X}=\{s \nrightarrow x \forall x \in X\}$ for each $X \subseteq V \backslash\{s\}$. Of course all these definitions collapse to those of Section 1 in case there are no oriented edges; so the next result contains Theorem 1.1.

Theorem 3.1. With the preceding modified definitions, Theorem 1.1 holds for directed percolation.

Proof. The proof is essentially the same as that of Theorem 1.1, the only difference being that we should now take $N$ to be the set of those $i \notin Z$ for which there is at least one edge $(i, j)$ or $\{i, j\}$ with $j \in Z$, and modify the definition of $S$ similarly.

Our first extension of Theorem 1.3 to directed percolation is

Theorem 3.2. Let $s \in V, X \subseteq V \backslash\{s\}$, and $f, g$ increasing functions of $C_{s}$. Then on $\{s \not X\}$,

$$
\mathrm{E} f g \geq \mathrm{E} f \mathrm{E} g
$$

This can be derived from Theorem 3.1 in the same way as Theorem 1.3 was derived from Theorem 1.1. It can also be proved using Markov chains (following either the proof of Theorem 2.1 or the alternate sketched afterwards), where we should now replace $C_{t}$ (in its various incarnations) by the set of edges in paths ending in $t$.

\section{Remarks, and consequences for Contact Processes}

(i) Analogously to what we said in Section 1, Theorem 3.2 can be stated in terms of (conditional) positive association; namely for any $X \subseteq V$, the random variables $\eta(y):=\mathbf{1}_{\{s \rightarrow y\}}$, conditioned on the event $\{\eta \equiv 0$ on $X\}$, are postively associated.

(ii) Taking $A=B=\Omega$ in Theorem 3.1 gives

$$
\operatorname{Pr}\left(R_{X}\right) \operatorname{Pr}\left(R_{Y}\right) \leq \operatorname{Pr}\left(R_{X \cup Y}\right) \operatorname{Pr}\left(R_{X \cap Y}\right) .
$$

(iii) Belitsky, Ferrari, Konno and Liggett ([2], Theorem 1.5) proved a special case of (17) involving a particular graph on the vertex set $\mathbb{Z}^{2}$. Their argument 
actually applies whenever $V$ admits a partition $\left(V_{0}=\{s\}\right) \cup V_{1} \cup \cdots$ such that each edge is directed from $V_{i-1}$ to $V_{i}$ for some $i$ and $X \cup Y$ is contained in some $V_{i}$, but does seem to depend essentially on these properties.

(iv) Much of [2] deals with the contact process on a countable set $S$. See [10] and [11] for background on this model; very briefly: Each site (individual) in $S$ can be in either of the states 1 (ill and contagious) or 0 (healthy, noncontagious). Time is continuous, with $\eta_{t}(x)$ denoting the state of site $x$ at time $t$. An infected site $x$ becomes healthy at rate $\delta_{x}$, and a healthy site becomes ill at rate $\sum_{y} \lambda(x, y) \eta(y)$. Here $\delta_{x}, x \in S$ and $\lambda(x, y), x, y \in S$, are the parameters of the model. They are assumed to be non-negative and, if $S$ is infinite, to satisfy the following conditions (see [2]): $\sup _{x \in S} \delta(x)<\infty$ and $\sup _{x \in S} \sum_{y \in S}[\lambda(x, y)+\lambda(y, x)]<\infty$.

A nice aspect of the model is that it can be viewed in terms of percolation, via a graphical representation (see e.g. [11], pages 32-34): being ill at some given time corresponds to the existence of an appropriate path in space-time. In fact, as is well-known, the process can, by time-discretization, be approximated by a directed percolation model. (See the subsection on correlation inequalities, in particular page 11, of [11] for the general idea of how correlation inequalities for collections of independent Bernoulli random variables can be extended to continuous-time interacting particle systems, and page 65 of [11] for a concrete example for the contact process). Combining this with the present results, one obtains, in a straightforward way, contact process analogues of the conditional association property stated in (ii) above. In particular this gives the following theorem.

Theorem 3.3. Suppose $\left(\eta_{t}: t \geq 0\right)$ is a contact process as above, with deterministic initial configuration $\eta_{0}$. Then for each $W \subset S$ and $t \geq 0$, the collection $\left(\eta_{t}(x): x \in S \backslash W\right)$ is conditionally positively associated given $\left\{\eta_{t} \equiv 0\right.$ on $\left.W\right\}$.

(An example of Liggett [12] shows that if we instead condition on $\left\{\eta_{t} \equiv 1\right.$ on $W\}$, the above positive association need not hold.)

Suppose now that at time 0 each site is ill. Let $\nu_{t}$ be the law of $\eta_{t}$ $\left(=\left(\eta_{t}(x): x \in S\right)\right)$. It is well-known (and follows easily from standard monotonicity arguments) that as $t \rightarrow \infty, \nu_{t}$ tends to a limit, called the upper invariant measure of the process and denoted $\nu$. Clearly the preceding conditional association property for finite times extends to $\nu$. So, if $W \subset S$, and $A$ and $B$ are events that are determined by, and that are both increasing 
(or both decreasing) in the $\eta(x), x \in S \backslash W$, then

$$
\nu(A B \mid \eta \equiv 0 \text { on } W) \geq \nu(A \mid \eta \equiv 0 \text { on } W) \nu(B \mid \eta \equiv 0 \text { on } W) .
$$

This is a considerable strengthening of a conjecture of Konno ([9], Conjecture 3.4.13), which was proved in - and seems to have been the main motivation for-[2] (see inequality (1.3) in [2]), namely: for any $K, L \subseteq S$,

$$
\nu(K \cap L) \nu(K \cup L) \geq \nu(K) \nu(L),
$$

where, for $M \subseteq S, \nu(M):=\nu\{\eta: \eta \equiv 0$ on $M\}$. (Of course (19) is the special case of (18) in which $W=K \cap L, A=\{\eta \equiv 0$ on $K \backslash L\}$ and $B=\{\eta \equiv 0$ on $L \backslash K\}$.)

\section{A directed version of Theorem 1.5}

For a sensible generalization of Theorems 1.4 and 1.5 to the present setting we need a different substitute for $\{s \nLeftarrow t\}$. It is easy to see that neither $\{s \not \rightarrow$ $t\}$ nor $\{s \nrightarrow t \nrightarrow s\}$ will do here (e.g. consider the graph on $\{s, t, v, a\}$ with (oriented) edges $(s, v),(t, v),(v, a)$, and events $A=\{s \rightarrow a\}, B=\{t \rightarrow a\})$; but there is another natural choice which does work, at least when we assume there are no undirected edges. Recall $V(F)$ is the set of vertices incident with edges of $F$.

Theorem 3.4. Assume $G$ is a digraph in the usual sense (that is, all its edges are directed). Let $s$ and $t$ be (distinct) vertices, and $f$ and $g$ bounded, measurable functions of $\left(C_{s}, C_{t}\right)$, each increasing in $C_{s}$ and decreasing in $C_{t}$. Then on $Q:=\left\{V\left(C_{s}\right) \cap V\left(C_{t}\right)=\emptyset\right\}$,

$$
\mathrm{E} f g \geq \mathrm{E} f \mathrm{E} g .
$$

As mentioned in Section 1, this can be proved along the lines of either Theorem 1.5 or Theorem 2.1, with Theorem 3.2 a crucial ingredient in either case. Here we only give (sketchily) the second argument, leaving the reader to fill in the first (which, like the second, depends on Observation 3.5 below).

It's a little strange that we can so far prove Theorem 3.4 only in the absence of undirected edges, and we conjecture that it remains true without this restriction. (The difficulties in extending the proof below-those for the other version are essentially the same - are the (related) failures of Observation 3.5 and of the validity of the hypothesis (iii) when we come to apply Lemma 2.6.) 
Proof. We will not repeat the proof of Theorem 2.1, but just indicate what changes are needed in the present situation.

The state space $\hat{\Omega}$ and transitions for our Markov chain are essentially as before. (Here we have chosen to say $C_{s}, C_{t}$ rather than $C_{S}, C_{T}$, but as noted earlier (following Theorem 1.2) this really makes no difference.) Of course $\left\{V\left(C_{s}\right) \cap V\left(C_{t}\right)=\emptyset\right\}$ now replaces $\{S \nLeftarrow T\}$ as the conditioning event $Q$.

Let us write $\psi$ for our (unconditioned) percolation measure, $\varphi$ for our $\psi$ conditioned on $Q$ and $\hat{\varphi}$ for the measure which $\varphi$ induces on $\hat{\Omega}$. The argument here then follows that for Theorem 2.1 verbatim until, in proving positive association of the collection (14), we come to establishing conditions (ii) and (iii) of Lemma 2.6. For these we need the easily verified (but crucial)

Observation 3.5. For $U \subseteq V$ the distribution of $C_{s}$ is the same under $\varphi$ conditioned on $\left\{V\left(C_{t}\right)=U\right\}$ as under $\psi$ conditioned on $\{s \nrightarrow U\}$ (and similarly with the roles of $s$ and $t$ reversed).

In view of this, (ii) is an immediate consequence of Theorem 3.2 (the relevant information from conditioning on $W$ being just the resulting value of $\left.C_{s}^{n-1}\right)$.

For (iii) we first observe that if $W, W^{\prime}$ are possible values of $\left(W_{1}, \ldots, W_{a}\right)$ with $W \leq W^{\prime}$, and $U, U^{\prime}$ are the corresponding values of $V\left(C_{s}^{n-1}\right)$, then according to Remark 2.8 we have $U \subseteq U^{\prime}$. Moreover, by Observation 3.5, the distribution of $\left(Z_{1}, \ldots, Z_{b}\right)\left(=\left(X_{e}^{n}: e \in E\right)\right)$ given $W$ is simply the distribution of (the indicator of) $E \backslash C_{t}$ under $\psi$ conditioned on $\{t \not t U\}$. So, writing $\hat{\varphi}_{U}$ for this distribution on $\left(X_{e}^{n}: e \in E\right)$, we need to show that $U^{\prime} \supseteq U$ implies $\hat{\varphi}_{U^{\prime}} \succ \hat{\varphi}_{U}$ (note that increasing $C_{t}^{n}$ corresponds to decreasing the $X_{e}^{n}$ 's). This follows from Theorem 3.2: Notice that $\hat{\varphi}_{U^{\prime}}$ is the same as $\hat{\varphi}_{U}$ conditioned on $B:=\left\{t \nrightarrow U^{\prime} \backslash U\right\}$. (More accurately, $\hat{\varphi}_{U^{\prime}}$ is the distribution induced on the indicator of $E \backslash C_{t}$ by $\psi(\cdot \mid t \not t)$ conditioned on $B$.) But then, since $B$ is a decreasing event determined by $C_{t}$, Theorem 3.2 says that under $\hat{\varphi}_{U}, B$ is negatively correlated with any increasing event determined by $C_{t}$; that is, $\hat{\varphi}_{U^{\prime}} \succ \hat{\varphi}_{U}$.

Remark. The choice of $\hat{\Omega}$ is a key to the preceding argument. For instance, taking the state space to be the analogue of that in the alternative proof of Theorem 2.1 sketched at the end of Section 2.1-namely $\left\{\omega \in\{0,1\}^{E}\right.$ : $Q$ holds for $\omega\}$ - gets in trouble because we lose some positive correlations, e.g. of events $\left\{\omega_{e}=1\right\}$. 
Acknowledgment We thank Tom Liggett for drawing our attention, after publication of our paper [3], to his paper [2] with Belitsky et al.

\section{References}

[1] R. Ahlswede and D.E. Daykin, An inequality for the weights of two families of sets, their unions and intersections, Z. Wahrsch. Verw. Geb. 43 (1978), 183-185.

[2] V. Belitsky, P.A. Ferrari, N. Konno and T.M. Liggett, A strong correlation inequality for contact processes and oriented percolation, Stoch. Proc. Appl. 67 (1997), 213-225.

[3] J. van den Berg and J. Kahn, A correlation inequality for connection events in percolation, Ann. Probab. 29, 123-126 (2001).

[4] B. Bollobás, Combinatorics, Cambridge Univ. Pr., Cambridge, 1986.

[5] L. Chayes, Percolation and ferromagnetism on $Z^{2}$ : the $q$-state Potts cases, Stoch. Proc. Appl. 65 (1996), 209-216.

[6] G. Grimmett, Percolation, 2nd edition, Springer, 1999.

[7] G. Grimmett, The random-cluster model, in Probability on Discrete Structures (H. Kesten, ed.), Encyclopedia of Mathematical Sciences, vol. 110, Springer (2003), 73-123.

[8] O. Häggström, Positive correlations in the fuzzy Potts model Ann. Appl. Probab. 9 (1999), 1149-1159.

[9] N. Konno, Phase Transitions of Interacting Particle Systems, World Scientific, Singapore, 1994.

[10] T.M. Liggett, Interacting Particle Systems, Springer, 1985.

[11] T.M. Liggett, Stochastic interacting systems: Contact, Voter and Exclusion Processes, Springer, 1999.

[12] T.M. Liggett, Survival and coexistence in interacting particle systems, Probability and Phase Transition, Kluwer, Dordrecht (1994), 209-226. 